\title{
La adolescencia: espacio de transformación
}

\section{Adolescence: A place for transformation}

\author{
Isabel Gonzalez Duarte \\ Psicologia LDA, Lisboa, Portugal
}

\begin{abstract}
Resumen: En este trabajo la autora se propone acceder al proceso que permite al niño convertirse en un adolescente. Se partirá de una inscripción en las teorías de la relación de objeto y en los modelos del pensamiento, explorando el espacio donde transcurren las transformaciones psíquicas que tienen lugar durante este período de desarrollo, permitiendo así, la construcción del sujeto de una forma única y singular. Tanto la noción de "procesos de transformación" reveladora de la dinámica mental presente durante este período de desarrollo, como con la noción de campo, generadora de encuentro, unidas a la techne, reveladora a su vez de la construcción, permiten una apertura para la comprensión de los procesos activos y dinámicos que se (re)construyen durante el proceso de conversión en adolescente. La clínica se presenta como un espacio privilegiado para la ilustración de las transformaciones, revelador de la relación intersubjetiva y de la co-construcción presentes en el convertirse adolescente.

Palabras clave: adolescencia, espacio, transformación, techne, campo.
\end{abstract}

\begin{abstract}
In this paper, the author proposes to access the process by which a child becomes an adolescent. The starting point will be an inscription in the object relations theories and the thought models, moving then to an exploration of the mental space where the mental transformations take place during this developmental period, allowing for construction of the subject in a unique, singular way. Both the concept of "transformation processes", revealing the mental dynamics of this developmental period, and the notion of field, generative of meeting, combined with the techne, in turn revealing construction, allow for openness for understanding the active, dynamic processes that are (re)built during the process of becoming an adolescent. The clinic represents a privileged space to illustrate the transformations, revealing the inter-subject relationship and the co-building involved in the process of becoming an adolescent.

Keywords: adolescence, mental space, transformation, techne, field.
\end{abstract}

Este artículo se basa en una presentación realizada en la $1{ }^{\text {a }}$ Conferencia Ibérica de Psicoanálisis Relacional: Espacios de Transformación que tuvo lugar en la III Jornadas de Psicoanálisis Relacional 5 Reunión Anual IARPP-España en Mayo de 2014 en Cáceres.

Para cualquier información sobre este artículo debe ponerse en contacto con la autora en el e-mail: isabelmgdc@gmail.com

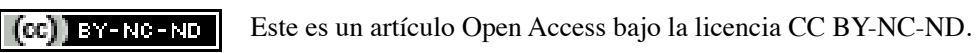


La adolescencia se caracteriza por ser el período de desarrollo durante el cual se produce el paso de la niñez a la edad adulta. Este proceso tiene lugar en un tiempo y se constituye como un espacio en el que existirán las transformaciones inherentes al crecimiento, ya que es el momento de renovar importantes funciones psicológicas, fundiéndose las estructuras que dan lugar a la formación de la identidad y a los procesos de identificación.

Durante años, se han llevado a cabo innumerables estudios sobre este período de desarrollo, comprobándose una extensión de los marcos teóricos, de una lógica más cercana a la psicopatología, que se entendía como un periodo en el que surgían sucesivas crisis y duelos, para pasar a comprenderse como un proceso de transformación y cambio, un espacio de cambio de/en cambio.

Me planteo pensar sobre el proceso de convertirse en adolescente. Para ello hago una inscripción en las teorías de la relación de objeto, en los modelos que nos permiten comprender los procesos de transformación y de co-construcción. Estos son conceptos que posibilitan conocer y dar a conocer el proceso de transformación, activo y dinámico, que es esencial para el crecimiento mental y es inherente al proceso de convertirse en adolescente. Este desarrollo se puede plantear como una relación de continente-contenido, a través de la cual el desconocido es de nuevo (re)significado, lo que permite la integración de los nuevos descubrimientos, e ir “aprendiendo con la experiencia" (Bion, 1962), en una relación intra e intersubjetiva, en y con lo(s) otro(s).

\section{Un Espacio en Construcción...}

Durante décadas muchos autores se han dedicado al estudio de la adolescencia. Las referencias más clásicas son realizadas bajo el modelo estructural, basado en que la pubertad da lugar a la aparición de las transformaciones que operan el paso de la vida sexual en su forma infantil a la forma adulta. Es de resaltar en dicho modelo que ocurre la construcción de las instancias psíquicas, del Yo y del Superyó, que son susceptibles de describir la tensión interna que conducen a nuevas relaciones del Yo y del Ello.

Más tarde, se comenzó a utilizar el modelo del duelo (Freud, 1917/1969), que fue basado en el análisis de la discontinuidad y de la necesidad de integración. Muchos de los trabajos desarrollados sobre la adolescencia, tienen por base las concepciones freudianas, incluso los realizados por Blos (1998) y Laufer (1980) que indican la existencia de pérdidas sucesivas, dando lugar a nuevas identificaciones.

Los enfoques más recientes nos informan sobre los procesos de transformación y construcción con base en los cuales es posible integrar lo nuevo aún desconocido. Braconnier (1985) propone el concepto de "procesos de transformación" para describir los procesos mentales que están en curso durante este período de desarrollo, en el cual existen mecanismos ya formados y otros aún en construcción.

Para Bollas (1987), la evocación de un objeto transformacional designa un funcionamiento psíquico y la representación de un objeto que permite la elaboración de las transformaciones internas. En la experiencia subjetiva primitiva, presente en la relación primaria, en la que el objeto de transformación puede ser asimilado a los procesos que modifican la experiencia.

Designa el primer objeto de transformador, donde describe el proceso presente en su relación con la experiencia de su anterior relación a la de la percepción de la madre como objeto completo. Considera la adolescencia como un "período de fragilidad" del crecimiento, dada la fuerte confrontación que se opera con el intenso dolor psíquico y el difícil dilema de incapacidad para llevar a cabo su simbolización, que implica la necesidad de crear un conjunto interno de emociones e ideas, que gestiona y autoriza la imaginación y la creatividad, funcionando como fuente de las relaciones interpersonales. Es la interiorización de la función del espejo y su uso en los diálogos intersubjetivos, como alternativa al juego interpersonal, que permite la adquisición de una capacidad introspectiva (Bollas, 2011).

El establecimiento de un espacio interno creador de nuevos sentidos y significados implica tener en cuenta las nociones de espacio y de tiempo. Matte-Blanco (1998) cuando hace referencia al espacio desarrolla una construcción matemática, que explica el espacio en abstracto, relacionándolo con la intuición. También sugiere que la noción de espacio se ha desarrollado basada en una reflexión sobre la experiencia perceptiva, pensando y avanzando hacia nuevas concepciones abstractas, quedando atrás nuestra percepción e imaginación. 
Con la teoría del pensamiento de Bion, tenemos un sistema de funcionamiento con espacio y tiempo, ya que interno y externo se consideran en interrelación, formulados a través de la dinámica continente-contenido, donde el continente y el contenido están unidos por la emoción y cambian de acuerdo con el crecimiento. Cuando se encuentran sin conexión emocional disminuye su vitalidad, es decir, se acercan a objetos inanimados (Bion, 1991). Si tenemos en cuenta la noción de espacio, entendida a la luz de estas concepciones, operamos un pasaje hacia el espacio interno capaz de contener y significar.

Para Cortiñas (2011) uno de los parámetros de crecimiento mental es la capacidad de tener visión y madurez para hacer frente a los conflictos emocionales, abriendo espacio para el desarrollo de pensamientos o utilizarlos para resolver problemas en ausencia de objetos. Sostiene que el crecimiento mental es atemporal y catastrófico, lo que nos lleva a la noción de cambio catastrófico y de clivaje, implicando la introducción de las nociones de espacio y de tiempo, como propulsoras del desarrollo de la capacidad de tolerar, impulsando a la creación de un espacio de "juego" que ofrece la posibilidad de modificar las ansiedades, favoreciendo el crecimiento.

Durante el proceso de convertirse en adolescente ocurren un conjunto de transformaciones psicológicas en el adolescente y en la relación que este establece con sus pares. En la adolescencia, edificarse a sí mismo sólo es posible cuando se establece una relación con lo(s) Otro(s) presentes durante este proceso de transformación, aquí se reflejan las igualdades, se acentúan las diferencias, favoreciendo el compartir, que genera un intercambio de complementariedades.

De este modo, se establece una dinámica interna, que Eiguer (2008) designó por la representación del sujeto y su relación con los demás, considerando fundamental la intersubjetividad para el desarrollo del afecto, permitiendo la construcción de otro lenguaje con un valor simbólico, un lenguaje corporal único y singular. El cuerpo psíquico contiene la representación de los objetos a los cuales el sujeto estuvo relacionado, por lo que no existe un cuerpo aislado, él está siempre en vínculo intersubjetivo, aunque primitivo o transformado en red de rasgos psíquicos.

Durante la adolescencia el cuerpo es un objeto de conocimiento, a la vez que sufre innumerables transformaciones se convierte, de nuevo en desconocido. Durante este período en el que se manifiesta el conflicto entre el cuerpo y la mente, se forman las bases para la estabilidad en la edad adulta. Sin embargo, este período se caracteriza por la turbulencia y la falta de tranquilidad, no en el sentido patológico, sino como un proceso de “aprendizaje por experiencia”, en el que la noción de desconocido de Bion, es de gran importancia para explicitar las transformaciones presentes en el proceso de convertirse en un adolescente (Lombardi, 2005).

Al final de la adolescencia, se hace posible establecer una relación con los padres como verdaderos objetos, dada la posibilidad de diferenciarse a sí mismo de los objetos (Carper, 1999). Los movimientos presentes oscilan entre el deseo de ser y el de estar en transformación, en alternancia entre las posiciones activas, donde domina la afirmación del sí mismo y las posiciones pasivas, marcadas por el retraimiento, cerrándose al nivel del Yo/Otro, una dificultad en conectarse entre lo interno y lo externo. El modelo de las transformaciones de Bion (1982) es el que mejor explica la relación de encuentro y comunicación entre el sujeto y el objeto y el objeto y el sujeto, entre lo interno y lo externo, entre el pasado y el presente, en una (re)creación de nuevos objetos con nuevas características y cualidades, permitiendo describir la circularidad del funcionamiento psíquico presente en lo que es convertirse en un adolescente.

La noción de campo desarrollada por Ferro (2011) se refiere a una función que se genera por el encuentro entre dos personas, a partir de la cual son promovidas narrativas, que no son más que la alfabetización de las proto-emociones presentes en la relación. El campo tiene en cuenta la importancia del funcionamiento mental, entendido en una lógica próxima a la que creó Bion (1970) “sin memoria ni deseo”. En el campo, lo importante no es cómo funciona el adolescente, sino más bien la posibilidad de observar la relación entre el funcionamiento y el disfuncionamiento, en el encuentro, en la relación. Es aquí donde se genera un campo donde es posible producirse la transformación. Se trata del encuentro donde los dos mundos se abren, como resultado de su encuentro, que no es apenas espacial, sino también temporal, en un continuo habitado por el presente y el pasado, abierto al futuro.

La adolescencia se caracteriza por ser un proceso de construcción de nuevos objetos y objetivos, de lo que se es y se desea ser, en un tiempo y en un espacio que es del propio. El concepto de techne nos permite pensar sobre los procesos en construcción presentes en el sujeto, ya que es un concepto que tiene en cuenta el proceso 
que permite la producción práctica de un objeto específico, que puede compararse con el “... trabajo de un escultor, que al comienzo no se siente seguro, pero que se va revelando durante el proceso de su creación" (Vassalli, 2001, p. 47), es como una improvisación de una tarea específica, que permite no sólo aprender con el problema, sino también tratar de darle solución. Para Caper (2009) es un tipo de aprendizaje cuya función es la de revelar el conocimiento. El adolescente que se convierte, es la obra de arte de sí mismo, siendo a la vez terapeuta y escultor, donde en el encuentro de la relación va a (re)crear el proceso de crecimiento y conocimiento. Así nace la obra de arte, resurgida del encuentro entre Uno y Otro, que el autor describe como una especie de aprendizaje, cuya función es la de revelar el conocimiento.

\section{EI Espacio para la Transformación...}

En el campo se genera el encuentro, revelador de la techne de Leonor, una adolescente de 13 años que llega a la consulta acompañada de sus padres. De ojos y pelo claro, alta y delgada, con una mirada expresiva busca la mirada del otro para situarse, en un intento de hacer frente a lo nuevo y desconocido del espacio.

Su madre es quien presenta a ambas, y esto manifiesta, antes de más, la falta de espacio que confirma el motivo que las trajo a la consulta, la existencia de la enuresis, sufrida por parte de su madre hasta la fecha de la primera regla y del padre hasta cerca de los 7 años. La madre describe que la enuresis no siempre es constante, tiene momentos en los que desaparece durante un período de tiempo, pero que más tarde, vuelve a reaparecer. En el pasado, ya habían buscado ayuda y Leonor estuvo siendo acompañada por una psicóloga, cuando tenía 9 y 10 años, por la misma razón. El síntoma desapareció y terminaron las consultas, pero los síntomas regresaron, revelando lo que era evidente, la falta de espacio para su transformación, a fin de convertirse en una adolescente.

Leonor estudia segundo de la ESO y ha suspendido 8 asignaturas en el primer trimestre. Aunque nunca haya sido una estudiante brillante, a pesar de las dificultades, siempre fue capaz de aprobar y pasar de curso. Su madre informa que el año pasado, la clase donde la habían colocado tuvo que ser divida en dos y a su hija le tocó frecuentar el turno del instituto por la tarde. Alejada de todos sus amigos, pasó los primeros meses llorando y diciendo que no quería ir más a clase. Sin embargo su madre pensó que no era correcto cambiarla, ya que si a todos los demás alumnos deseasen también cambiar el turno, eso sería insoportable. Esta es la justificación dada por la madre, sin ningún tipo de eco emocional por lo sucedido con su hija.

La enuresis infantil es una constante; sin embargo fue una niña sana, sin haber tenido las enfermedades más comunes en la infancia. Recientemente se quejó que oía mal, y la llevaron a hacerse un audiograma, revelando una Otoesclerosis, que causará una pérdida de audición muy ligera, existiendo la necesidad de realizar una pequeña cirugía correctiva. Este es un problema de orden genético ya que su padre también sufre del mismo problema y tuvo que ser sometido a una intervención para corregirlo.

El contacto para la consulta se le había dado a la madre a quien se le diagnosticó el marco de la bipolaridad y que sigue una terapia con medicamentos y sesiones psicológicas con otra psicóloga. Los espacios se interrelacionan, es evidente el fallo relacional, la dificultad en constituir un espacio de construcción donde puedan ocurrir las transformaciones, donde se puedan "escuchar" los miedos, las ansiedades, los temores presentes en el conturbado proceso de desarrollo que está atravesando.

Leonor explica que se siente muy avergonzada de esta situación, que a su juicio, está directamente relacionada con lo que sucede en el instituto, donde un grupo de colegas se ríen a menudo de ella, y les oye decir: "qué asco". Al respecto afirma: "Yo sé que ellos no lo saben, pero tengo demasiado miedo que lo descubran". La falta de espacio sentido en la relación familiar, le dificulta situarse en su grupo de pares.

En una de las primeras sesiones describe un sueño que tuvo, el material para la realización de la obra de arte, generando el encuentro creativo, la mejor forma de revelarlo. "Yo soñé con un ejército, había un camino en el medio, y sonó un timbre y comenzó una pelea, y yo me encontraba allí en el medio sin entender qué es lo que estaba haciendo allí.” Aquí presentamos lo que describe Matte-Blanco (1998), la existencia de una relación lógica entre dos pensamientos, experimentada en los sueños, colocándolos uno a la par 
del otro, en una relación espacial y por lo tanto pudiendo ocurrir a la vez, llevando a la desaparición de la sucesión temporal.

Este sueño es un boceto en el encuentro, en la relación, de un espacio para la transformación. Dos fuerzas opuestas, la materna y la paterna, una indiferenciación generadora de conflicto inherente al propio proceso de llegar a ser, durante el cual las diversas partes dispersas deben reunirse, conectarse e integrarse entre sí. El timbre, estrechamente relacionado con el mundo externo, el instituto, pero también el mundo interior, el eco de la alarma, despertando para nuevos deseos y retos, autorizándose, liberándose de la prisión en la que ha vivido, impedida de crecer y de ser.

El encuentro psicoterapéutico es marcado por una enorme falta de espacio, en el discurso de Leonor es notoria la indiferenciación entre si y los demás(s) en particular, su madre, con quejas frecuentes, sintiendo el malentendido entre sus deseos y aspiraciones, no reconociendo su singularidad y su individualidad, encontrándose su Yo, todavía muy frágil.

De espacio, Leonor se dará cuenta de cómo se siente asfixiada en su relación con su madre. No es posible hacerse oír y entender, suscitando una descarga física durante la noche, la enuresis, muchas veces acompañada por sueños, en los cuales es inmediatamente evidente la necesidad de tener una voz, para hacerse comprender, tener un lugar donde existir en su familia y en el mundo.

El padre es una figura poco presente en la dinámica familiar. Leonor describe a su padre como muy trabajador y muy ocupado, poco disponible para sus necesidades. La vida social de la familia es muy activa, se suceden las fiestas, los eventos, las reuniones, para los cuales Leonor sigue siendo arrastrada sin espacio para que sus intereses, o sus necesidades, sean oídas.

Leonor ha suspendido el curso. Este acontecimiento ha sido muy importante en el contexto de la terapia, porque de este modo ha sido posible separar la realidad de la fantasía. Mediante el evento de la realidad, Leonor ha tenido consciencia de la necesidad de formar su propio espacio, en la realidad externa, en la nueva escuela, un nuevo espacio, un cambio vivido con una mezcla de ansiedad y deseo, que en la psicoterapia ha llevado a la construcción de un espacio interno. En su construcción mental finalmente emergía un boceto de un lugar para pensar en la relación con lo(s) Otro(s).

Estamos en el segundo año de la psicoterapia, con una frecuencia semanal, cuando en una sesión, a propósito del baile de finalistas, que tendrá lugar en las próximas semanas, Leonor se muestra muy indignada al inicio de la sesión: "Estuve discutiendo con mi madre... no quiero que vaya a mi baile jes mi baile! Ella dice que no tiene importancia... es horrible, como puede hacerme esto...”. Ha sido la posibilidad de ir construyendo su Yo, en el campo del espacio psicoterapéutico, que hace que Leonor reconozca las constantes invasiones de su madre.

Trabajamos juntas la necesidad de delimitar su espacio, de tener voluntad propia y, por primera vez, le fue posible a Leonor expresar su deseo, contradictorio al de su madre. Estaba cada vez más clara la necesidad de tener su propio espacio, diferenciado de los demás, empieza a ser posible llevar consigo el espacio intersubjetivo y de comunicación construido en las sesiones.

Pero su madre no respetó su deseo y fue al baile. La ida de su madre a su baile fue vivida con una mezcla de invasión y de vergüenza. Leonor presenta una postura crítica sobre el comportamiento desajustado de su madre y por primera vez verbalizado, comenzando la enuresis finalmente a ceder.

Ha pasado un año y Leonor está en la recta final de su adolescencia. En este momento se enfrenta a una situación dolorosa, el cambio de domicilio. Sus padres han perdido la casa por las deudas acumuladas en la empresa. Su nueva casa es un espacio ubicado en la planta baja de la vivienda de su abuela paterna. Tendrá que compartir habitación con su hermana, algo distinto a lo vivido hasta el momento, pues siempre tuvo su propia habitación. Su madre ha tenido una idea bizantina, ponerle un mueble en medio de la habitación para dividir el espacio, lo que Leonor ha negado, comportándose de forma más adulta: “...si voy a tener que compartir mi habitación, que éste sea un espacio agradable para mí y para mi hermana.”

Más segura de su espacio mental y relacional, Leonor decide participar en un intercambio con otra estudiante en el extranjero. Este es un momento particularmente doloroso en la terapia de Leonor, por la toma de consciencia de la incapacidad de transformación de su familia después de confrontarla con realidades tan distintas de otras familias. Ahora hay espacio para vivir y soñar por su propia iniciativa. 
En la recta final de su proceso de convertirse en adolescente, el trabajo psicoterapéutico se ha centrado en la necesidad de sacar buenas notas e ir a la Universidad. Esta es una posibilidad real de apartarse de su familia. Mi función como psicoterapeuta, ha sido el de hacer funcionar como un campo, soportando su autonomía, desarrollando un espacio interior en el cual sea posible reflexionar sus propios deseos y emociones, los cuales son al final presentados de forma creativa.

\section{Un Espacio (In)Concluso}

Ser un adolescente constituye un imperativo en la construcción de un espacio psíquico abierto al conocimiento, facilitador del crecimiento por lo que debe ser contenedor y transformador de las funciones psíquicas que se estructuran durante este período en la relación Yo/Otro.

Explicitar el espacio de/en transformación actual para convertirse en adolescente, implica el uso de un amplio marco teórico que permita la apertura de pensamiento, lo que constituye un valor añadido en la práctica clínica, transformando el encuentro pensando como siendo un campo (Ferro, 2011) potenciador de la techne (Vassalli, 2001), un espacio de construcción, generador de nuevo(s) sentido(s) y significado(s).

El encuentro clínico deberá entenderse como un espacio revelador del hacerse adolescente, siendo de suma importancia, dado su contexto relacional, potenciador de creatividad. Pero también, como un espacio de/en cambio, en el cual se puede (re)construir espacios, delimitadores del Yo y del Otro, revelando la singularidad del adolescente, presente en la intersubjetividad y en el espacio de co-construcción.

\section{Referencias}

Bion, W. R. (1962). Learning from experience. Londres, Reino Unido: Karnac.

Bion, W. R. (1970). Atenção e Interpretação. O acesso científico à intuição em psicanálise e grupos. Rio de Janeiro, Brasil: Imago Editora.

Bion, W. R. (1982). As transformações. A mudanças do aprender para o crescer. Rio de Janeiro, Brasil: Imago Editora.

Bion, W. R. (1991). As transformações. As mudanças do aprender para o crescer. Rio de Janeiro, Brasil: Imago.

Braconnier, A. (1985). Ruptures et séparations. Adolescence, 3(1), 5-19.

Blos, P. (1998). Adolescência. Uma interpretação Psicanalítica. São Paulo, Brasil: Martins Fontes.

Bollas, C. (1987). The Shadow of the object. Psychoanalysis of the unthought known. Londres, Reino Unido: Free Association Book.

Bollas, C. (2011). The Cristopher Bollas Reader. Nueva York, Estados Unidos: Routledge.

Caper, R. (1999). A mind of One's Own. A Psychoanalytic View of Self and Object. Londres, Reino Unido: The New Library of Psychoanalysis.

Caper, R. (2009). Building out into the dark. Theory and observation in science and psychoanalysis. Nueva York, Estados Unidos: Routledge.

Cortiñas, L. P. (2011). Science and fiction in the psychoanalytical field. In Mawson, C. (Ed). Bion Today, 121152. Nueva York, Estados Unidos: Routledge.

Eiguer, A. (2008). Jamais moi sans toi. Paris, Francia: Dunod.

Ferro, A. (2011). Avoiding Emotions, Living Emotions. Nueva York, Estados Unidos: Routledge.

Freud, S. (1969). Luto e Melancolia. En Edição Standart Brasileira das Obras Psicológicas Completas de Sigmund Freud Vol. XVIII. Rio de Janeiro, Brasil: Imago Editora. (Obra original publicada en 1917).

Laufer, M. (1980). L'idéal du moi et le pseudo-idéal du moi a l'adolescence. Revue Française de Psychanalyse. 3, 591-615. 
Lombardi, R. (2005). From the eclipse of the body to the dawn of thought. International Journal of Psycho-Analysis, 86, 579-585.

Matte-Blanco, I. (1998). Thinking, Feeling and Being. Clinical reflections on the fundamental antinomy of human beings and world. Londres, Reino Unido: Routledge.

Vassalli, G. (2001). The birth of Psychoanalysis from the spirit of Technique: what have we learned? How can we Apply it? International Journal of Psycho-Analysis, 82, 3-23.

Artículo recibido: 29/04/2016

Revisión recibida: 09/01/2017

Artículo aceptado: 25/01/2017 